\title{
Phonon transport in periodic silicon nanoporous films with feature sizes greater than $100 \mathrm{~nm}$
}

\author{
Ankit Jain, Ying-Ju Yu, and Alan J. H. McGaughey* \\ Department of Mechanical Engineering, Carnegie Mellon University, Pittsburgh, Pennsylvania 15213, USA
}

(Received 4 December 2012; revised manuscript received 1 February 2013; published 3 May 2013)

\begin{abstract}
The thermal conductivities of solid silicon thin films and silicon thin films with periodic pore arrays are predicted using a Monte Carlo technique to include phonon-boundary scattering and the Boltzmann transport equation. The bulk phonon properties required as input are obtained from harmonic and anharmonic lattice dynamics calculations. The force constants required for the lattice dynamics calculations are obtained from forces calculated using density functional theory. For both solid and porous films, the in-plane thermal conductivity predictions capture the magnitudes and trends of previous experimental measurements. Because the prediction methodology treats the phonons as particles with bulk properties, the results indicate that coherent phonon modes associated with the secondary periodicity of the pores do not contribute to thermal transport in porous films with feature sizes greater than $100 \mathrm{~nm}$.
\end{abstract}

DOI: 10.1103/PhysRevB.87.195301

PACS number(s): 63.20.-e, 63.22.-m, 65.80.-g, 66.70.-f

\section{INTRODUCTION}

Experimental studies on silicon thin films with a periodic arrangement of unfilled cylindrical pores (i.e., nanoporous films; see Fig. 1) measured thermal conductivities two orders of magnitude smaller than bulk silicon. ${ }^{1-3}$ Hopkins et al. studied four 500-nm-thick films with a square array of pores with diameters and pitches between 300 and $800 \mathrm{~nm}$, finding cross-plane (i.e., parallel to the pore axis) thermal conductivities between 5 and $7 \mathrm{~W} /(\mathrm{m} \mathrm{K})$ at a temperature of $300 \mathrm{~K} .{ }^{3}$ Yu et al. studied a $22-\mathrm{nm}$-thick film with a square array of pores with diameter of $11 \mathrm{~nm}$ and pitch of $34 \mathrm{~nm}$, finding an in-plane (i.e., normal to the pore axis) thermal conductivity of 2 $\mathrm{W} /(\mathrm{m} \mathrm{K})$ that did not vary significantly between temperatures of 150 and $280 \mathrm{~K} .^{1}$ Tang et al. studied a 100 -nm-thick film with a triangular array of pores with diameter of $32 \mathrm{~nm}$ and pitch of $55 \mathrm{~nm}$, finding an in-plane thermal conductivity of $2 \mathrm{~W} /(\mathrm{m} \mathrm{K})$ at a temperature of $300 \mathrm{~K} .^{2}$ Such low thermal conductivities, combined with predictions of bulk-like electrical transport properties, ${ }^{4}$ suggest the potential application of nanoporous films in thermoelectric energy conversion.

The low thermal conductivities of these silicon nanoporous films, in which phonons dominate thermal transport, cannot be predicted by considering thermal conductivity reduction due to material removal and the presence of phonon-boundary scattering. ${ }^{3,5}$ Based on the modeling work of Hao et al., ${ }^{6}$ Tang et al. suggest a "necking effect," whereby phonons with mean free paths longer than the distance between two pores (i.e., the neck, equal to the pitch minus the diameter) become "trapped" behind pores. Yu et al. and Hopkins et al. suggest that the low thermal conductivities are a result of "coherent phonon effects," whereby the phonon band structure and scattering are modified by the secondary periodicity introduced by the pores.

More recently, El-Kady et al. ${ }^{7}$ reported in-plane thermal conductivity measurements for nanoporous films similar to those studied by Hopkins et al. They found thermal conductivities between 33 and $80 \mathrm{~W} /(\mathrm{m} \mathrm{K})$, an order of magnitude larger than the cross-plane results reported by Hopkins et al. For a 500-nm-thick solid silicon film, modeling work suggests that the in-plane thermal conductivity will only be $15 \%$ larger than the cross-plane value. ${ }^{8}$ Furthermore, the size of the structural features and the magnitudes of the thermal conductivities measured by El-Kady et al. are similar to previous experimental work on solid films and nanowires. ${ }^{9-11}$ Phonon particle-based models that use bulk properties are able to capture the experimental thermal conductivity trends for these films and nanowires by including phonon-boundary scattering. $8,9,11,12$

In this work, we will predict the thermal conductivity of solid silicon films and silicon nanoporous films with feature sizes greater than $100 \mathrm{~nm}$ using the phonon free path sampling technique developed by McGaughey and Jain. ${ }^{13}$ This technique treats the phonons as particles with bulk properties that are modified by phonon-boundary scattering. As such, any effects of the secondary periodicity on the phonon transport, other than the geometry for the boundary scattering, are excluded. Thus, we can determine if coherent effects play a role in phonon transport in the nanoporous films studied by Hopkins et al. and El-Kady et al. Furthermore, this technique includes Bose-Einstein phonon statistics (i.e., quantum effects), considers the full phonon spectrum, is computationally fast, and can be applied to three-dimensional structures with arbitrary geometry. To ensure an accurate comparison with the experimental measurements, phonon properties obtained from forces calculated using density functional theory (DFT) ${ }^{14,15}$ are used as input, removing the need for empirical force fields or fitting to experimental data.

\section{PREVIOUS MODELING WORK}

Yang et al., ${ }^{16}$ Prasher, ${ }^{17}$ and Romano et al. ${ }^{18}$ solved the Boltzmann transport equation (BTE) under the gray approximation (i.e., frequency-independent phonon properties) for periodic nanoporous films with a square array of cylindrical pores. They all considered the scattering of phonons with other phonons and pore boundaries and found that the inplane thermal conductivity depends not only on porosity, but also on pore size and pitch. Hao et al. used Monte Carlo simulations to study in-plane phonon transport in periodic silicon nanoporous films with a square array of square pores, including frequency-dependent phonon properties. ${ }^{6}$ Including this frequency dependence is critical, since recent modeling work has demonstrated the importance of including mode-dependent phonon properties in calculating the thermal 


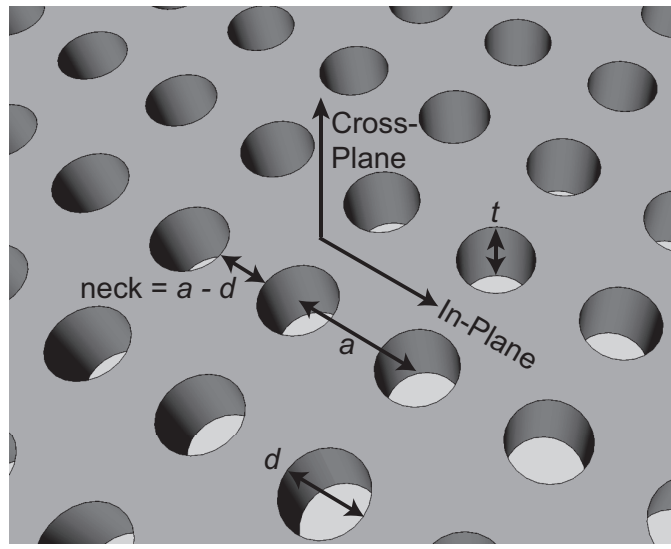

FIG. 1. Thin film with a square array of through cylindrical pores.

conductivity of bulk and nanostructured materials (e.g., the phonon mean free paths in bulk silicon cover five orders of magnitude). ${ }^{15,19,20}$ In these numerical and analytical models, the phonon modes that emerge due to the pore periodicity were not included. Moreover, these studies considered twodimensional geometries and therefore cannot account for phonon scattering from the top and bottom film boundaries or allow for examination of cross-plane thermal transport.

He et al. ${ }^{21}$ used molecular dynamics (MD) simulations and lattice dynamics calculations to predict the in-plane thermal conductivity of periodic silicon nanoporous films with diameters and pitches less than $10 \mathrm{~nm}$. By separating the vibrational modes into phonons and diffusons (i.e., nonpropagating, diffusive entities) and including pore surface roughness, they found thermal conductivities as much as 70 times smaller than bulk. For the same geometry but with smaller diameters and pitches, Lee et al. used MD simulations to predict thermal conductivities more than 100 times smaller than bulk. ${ }^{22}$ Molecular dynamic simulations have the advantage of naturally capturing the wave nature of phonons, but are limited to system sizes less than $100 \mathrm{~nm}$ due to computational cost. They therefore cannot be used to model the films of Yu et al., Tang et al., Hopkins et al., or El-Kady et al. Furthermore, MD simulations cannot incorporate Bose-Einstein statistics in the phonon populations.

Dechaumphai and Chen define "coherent" phonons to be those with mean free paths larger than the neck. ${ }^{5}$ These phonons are modeled using the in-plane dispersion relation for the periodic pores. They scatter with other phonons, but not from the pores. Phonons with mean free paths shorter than the neck are termed "incoherent," treated as bulk-like, and scatter with other phonons and the pores. Dechaumphai and Chen predict that their coherent phonons will contribute $\sim 1 \mathrm{~W} /(\mathrm{m} \mathrm{K})$ (i.e., $\sim 50 \%$ ) to the in-plane thermal conductivity of the nanoporous films of Yu et al. and Tang et al., but only $0.01 \mathrm{~W} /(\mathrm{m} \mathrm{K})$ (i.e., less than $0.1 \%$ ) to the structures of Hopkins et al. and El-Kady et al. Using a similar approach for the phonon dispersion, Reinke et $a .^{23}$ modeled the nanoporous films of El-Kady et al., but do not differentiate in how "coherent" and "incoherent" phonons scatter. They find similar trends compared to the experimental data in thermal conductivity versus neck size and pore pitch, but their predicted magnitudes are $\sim 15 \mathrm{~W} /(\mathrm{mK})$ too high. Hopkins et al. ${ }^{3}$ find reasonable agreement with their cross-plane experimental measurements using a similar approach as Reinke et al., with the in-plane phonon dispersion applied to the cross-plane transport.

\section{METHODOLOGY}

The phonon contribution to thermal conductivity in the $n$ direction is given by

$$
k_{n}=\sum_{i} c_{p h, i} v_{g, n, i}^{2} \frac{\overline{\Lambda_{i}}}{\left|\mathbf{v}_{g, i}\right|},
$$

an expression obtained by solving the BTE under the relaxation time approximation and using the Fourier law. The summation is over all the phonon modes (indexed by $i$ ) in the first Brillouin zone. On the right-hand side, $c_{p h, i}$ is the volumetric phonon specific heat, $v_{g, n, i}$ is the $n$ component of the phonon group velocity vector $\mathbf{v}_{g, i}$, and $\overline{\Lambda_{i}}$ is the phonon mean free path, defined as the average distance traveled by a phonon before it scatters. The phonon volumetric specific heat is calculated using Bose-Einstein statistics. The phonon group velocity vector is $\mathbf{v}_{g, i}=\partial \omega_{i} / \partial \boldsymbol{\kappa}$, where $\omega$ is the phonon frequency and $\boldsymbol{\kappa}$ is the phonon wave vector. Lattice dynamics calculations can be used to predict bulk phonon frequencies and intrinsic mean free paths (i.e., corresponding to phonon-phonon scattering) in a framework that includes Bose-Einstein statistics. ${ }^{15,24-27}$

The standard approach for including phonon-boundary scattering in the phonon mean free path is to use the Matthiessen rule, ${ }^{28,29}$ whereby

$$
\frac{1}{\overline{\Lambda_{i}}}=\frac{1}{\overline{\Lambda_{p p, i}}}+\frac{1}{\overline{\Lambda_{p b, i}}} .
$$

Here, $\overline{\Lambda_{p p, i}}$ and $\overline{\Lambda_{p b, i}}$ are the phonon mean free paths corresponding to scattering from other phonons and from system boundaries. The Matthiessen rule assumes that different phonon scattering mechanisms are independent. The phonon-phonon mean free path is typically taken from bulk calculations. The phonon-boundary mean free path is typically based on the Casimir limit, ${ }^{29,30}$ which can be difficult to obtain for an arbitrary nanostructure geometry.

The effective mean free path calculated using the Matthiessen rule is based on mean values of the phononphonon and phonon-boundary free paths. Because of the nonlinear nature of the Matthiessen rule, it is not clear if this effective mean free path will be the same as that obtained when the natural distributions of phonon-phonon and phonon-boundary free paths are considered.

To remove the aforementioned assumptions and ambiguities in the application of the Matthiessen rule, the free path sampling technique developed by McGaughey and Jain ${ }^{13}$ is used in this study. As with the Matthiessen rule, bulk phonon properties are assumed to be valid in the nanostructure. Turney et al. suggest that using bulk phonon properties to model suspended silicon films is valid for limiting dimensions (i.e., film thicknesses) greater than $20 \mathrm{~nm} .{ }^{31}$ In a nanoporous film, the limiting dimension is the lesser of the film thickness and the neck. As such, the free path sampling technique can be applied to the nanoporous films studied by Hopkins et al. and 
El-Kady et al., which have limiting dimensions of at least $200 \mathrm{~nm}$. The free path sampling technique cannot be applied to the nanoporous films of Yu et al. (limiting dimension $22 \mathrm{~nm}$ ) or Tang et al. (limiting dimension $23 \mathrm{~nm}$ ).

The thermal conductivity prediction proceeds as follows: first, a free path corresponding to phonon-phonon scattering is selected from a Poisson distribution whose average value is the bulk mean free path. Second, the initial position of the phonon is sampled uniformly inside the nanostructure volume. These two assumptions have been verified for symmetric nanostructures using an energy-based deviational formulation for solving the BTE. ${ }^{32,33}$ The distance from the initial position to the structure boundary in the direction of the group velocity vector is the sampled phonon-boundary free path. The minimum of these two sampled free paths is the effective phonon free path. That is,

$$
\Lambda_{i}=\min \left(\Lambda_{p p, i}, \Lambda_{p b, i}\right) .
$$

In this way, the assumption of the independence of the scattering mechanisms made in the Matthiessen rule is removed. This process is repeated for each phonon mode until the average effective free path converges to the mean free path (1000 samples per phonon mode in this study, which leads to an uncertainty of less than $1 \%$ in the final thermal conductivity prediction $^{13}$ ). Given a set of bulk phonon properties, each thermal conductivity prediction takes a few seconds.

Diffuse boundaries are assumed for the phonon-boundary scattering. Modeling work by Duda et al. suggests that all phonon-boundary scattering will be diffuse at a temperature of $300 \mathrm{~K}^{34}$ The scattering of phonons by point defects is ignored. Previous experimental measurements on bulk silicon indicate that the effect of phonon-isotope scattering is small compared to phonon-phonon scattering at a temperature of $300 \mathrm{~K} \cdot{ }^{35}$ The doping concentration of the experimental samples that we compare against is $10^{17} \mathrm{~cm}^{-3}$ or lower. Previous experimental measurements indicate that dopant atoms at these concentrations do not significantly affect the thermal conductivity of silicon at a temperature of $300 \mathrm{~K}^{36,37}$

Based on the two-atom (i.e., primitive) unit-cell description, harmonic and anharmonic lattice dynamics calculations ${ }^{15,24-27}$ are performed to calculate the bulk silicon phonon frequencies and mean free paths for an $18 \times 18 \times 18$ grid of uniformly spaced wave vectors centered at the origin of the Brillouin zone. 34992 total phonon modes are considered, which includes acoustic and optical branches. The second- and thirdorder force constants are those obtained by Esfarjani et al. from forces calculated using DFT and a numerical approach that enforces the required symmetries. ${ }^{14,15}$ Bose-Einstein statistics at a temperature of $300 \mathrm{~K}$ are used to incorporate quantum effects.

\section{THERMAL CONDUCTIVITY PREDICTIONS}

\section{A. Bulk silicon}

Using Eq. (1), the bulk thermal conductivity of silicon is predicted to be $132 \mathrm{~W} /(\mathrm{m} \mathrm{K})$, while the experimental value for isotopically enriched silicon is $144 \mathrm{~W} /(\mathrm{m} \mathrm{K}) .^{35}$ Due to the finite resolution of the Brillouin zone, the contributions of phonons in the volume at the $\Gamma$ point are not included in the calculation. Following the approach described by Esfarjani et al., ${ }^{15}$ we estimate the contribution of these phonons to

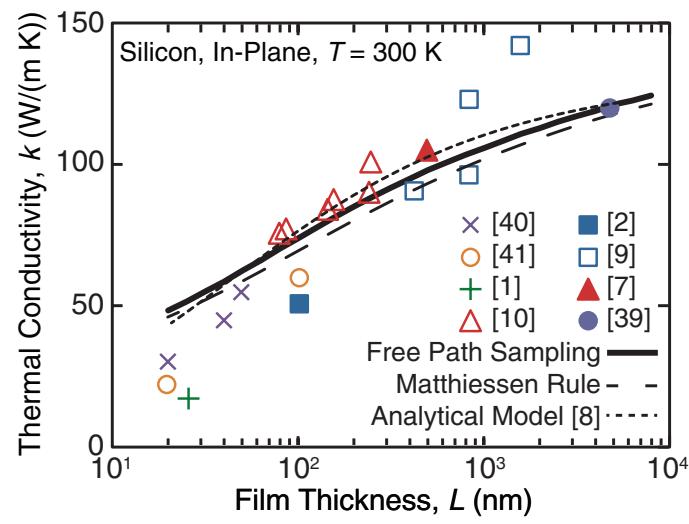

FIG. 2. (Color online) In-plane thermal conductivity of solid silicon thin films vs film thickness at a temperature of $300 \mathrm{~K}$ from the free path sampling technique, the Matthiessen rule, an analytical model, and experiments. For the analytical model, we take the sound speed to be $6733 \mathrm{~m} / \mathrm{s}$ and the lattice constant to be $5.43 \mathrm{~A} \cdot{ }^{42}$ The bulk thermal conductivity in the analytical model is set to $132 \mathrm{~W} /(\mathrm{m} \mathrm{K})$ to be consistent with the value found in Sec. IV A.

be $10 \mathrm{~W} /(\mathrm{m} \mathrm{K})$. Based on the work of Broido et al., ${ }^{38}$ a further $10 \%$ increase in the thermal conductivity of silicon is expected if one solves the BTE iteratively without making the relaxation time approximation, making the predicted bulk thermal conductivity $156 \mathrm{~W} /(\mathrm{m} \mathrm{K})$. This value is higher than the experimental measurement because of the complete absence of point defects, which will be present in any real samples, and uncertainty associated with the DFT calculations. For our purposes, the relaxation time approximation is needed as solving the BTE iteratively does not provide the mean free paths required to predict nanostructure thermal conductivity.

\section{B. Solid thin films}

To validate the free path sampling technique and the use of bulk phonon properties for modeling nanostructures, we first consider solid silicon thin films that have finite thickness in one direction and are infinite in the other two directions. The predicted thermal conductivity variation with film thickness $L$ for heat flow in the in-plane direction obtained using the free path sampling technique and the Matthiessen rule are shown in Fig. 2 with available experimental data. ${ }^{1,2,7,9,10,39-41}$ The experimental data include the solid films of Yu et al., Tang et al., and El-Kady et al. The thermal conductivity predictions from the Matthiessen rule are obtained using

$$
\overline{\Lambda_{p b, i}}=\frac{L}{2 \cos \theta_{i}},
$$

where $\theta_{i}$ is the angle between the cross-plane direction and the group velocity vector. ${ }^{8}$

Also plotted in Fig. 2 are the predictions from a closedform analytical model. ${ }^{8}$ This model is based on the Debye approximation for the phonon dispersion and ignores the contribution of optical phonons to thermal conductivity. The lattice constant, a sound speed, and the bulk thermal conductivity are the required inputs.

The thermal conductivity increases with increasing film thickness due to a reduction in phonon-boundary scattering. 
The free path sampling technique, the Matthiessen rule approach, and the analytical model capture the experimental magnitudes and trend well for thicknesses greater than $50 \mathrm{~nm}$. This transition point is higher than the $20 \mathrm{~nm}$ suggested by Turney et al., ${ }^{31}$ which was based on the density of states and in-plane thermal conductivity of suspended silicon thin films modeled using the Stillinger-Weber potential. The finite simulation size and relaxation time approximation errors are smaller in thin films compared to bulk due to the scattering of long mean free path phonons from film boundaries, which reduces their contribution to the thermal conductivity.

\section{Periodic nanoporous films \\ 1. In-plane direction}

The periodic nanoporous films of interest have three characteristic length scales: pore diameter $d$, pore separation $a$, and film thickness $t$, as shown in Fig. 1. It is not obvious what length scale is appropriate in the Matthiessen rule for these structures. We therefore use the free path sampling technique to predict their thermal conductivities. To allow for comparison with the experimental data, the predicted thermal conductivities must be scaled by a volume correction factor to account for the porosity $\phi$. For the in-plane direction, the correction factor is calculated using the COMSOL Multiphysics ${ }^{\circledR}$ simulation package to solve the heat conduction equation with the finite element method (FEM). Our FEM calculations agree with the correction factor proposed by Hashin and Shtrikman, $(1-\phi) /(1+\phi),{ }^{43}$ to within $1 \%$. The Eucken factor, $(1-$ $\phi) /(1+2 \phi / 3),{ }^{44}$ overpredicts the FEM calculations with a deviation that increases with increasing porosity. For a porosity of 0.4 , the maximum considered here, the deviation is $10 \%$. The volume correction factor is $1-\phi$ for the cross-plane direction.

The thermal conductivity variation with porosity for inplane heat flow in 500-nm-thick nanoporous films predicted using the free path sampling technique is shown in Fig. 3(a). The pore separations (400-900 nm) and diameters (200$500 \mathrm{~nm}$ ) are chosen to match the nanoporous films studied by El-Kady et al. The experimental thermal conductivity measurements of El Kady et al. are also plotted in Fig. 3(a). Both the experimental measurements and modeling predictions show a general decrease in thermal conductivity with increasing porosity because of more phonon-pore scattering and a reduced solid fraction. The maximum error between the experimental and the predicted thermal conductivities is $6 \%$ (corresponding to the solid film, where $\phi=0$ ).

At a porosity of around 0.15 in Fig. 3(a), measurement and modeling results show an increase in thermal conductivity with increasing porosity. To understand this result, we plot thermal conductivity as a function of pore diameter for a fixed porosity of 0.15 in the inset of Fig. 3(a). For this porosity, a thermal conductivity variation of up to $9 \%$ can be obtained by varying the pore diameter across the range of diameters considered. This finding is similar to what Yang et al. observed ${ }^{16}$

The predicted thermal conductivities are based on the bulk phonon dispersion and do not take into account changes to the phonon band structure due to the pore periodicity. Based on the good agreement between the measured and predicted
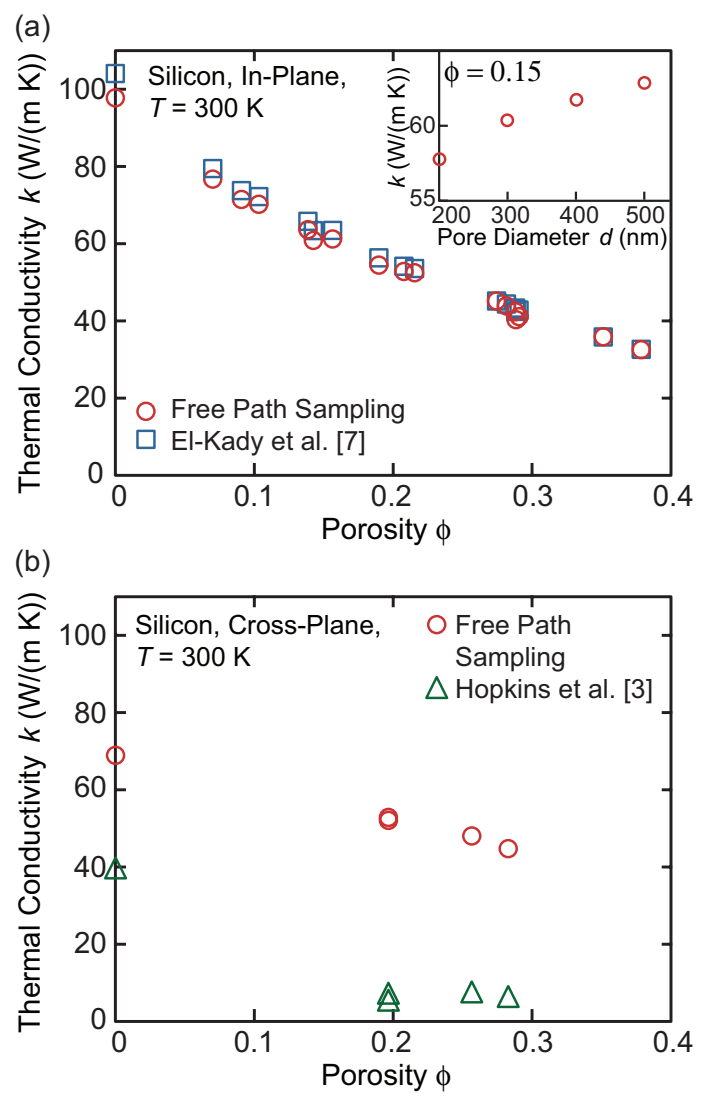

FIG. 3. (Color online) Thermal conductivity variation of periodic nanoporous silicon films vs porosity from experimental measurements and modeling predictions for (a) in-plane heat flow, and (b) cross-plane heat flow.

thermal conductivities, we do not believe that coherent phonon effects affect thermal transport in the nanoporous films of El Kady et al.

We also predicted the in-plane thermal conductivity of the nanoporous film studied by Song and Chen, ${ }^{39}$ which had thickness $4.67 \mu \mathrm{m}$, pore diameter $1.9 \mu \mathrm{m}$, and pore pitch $4 \mu \mathrm{m}$, to be $63 \mathrm{~W} /(\mathrm{m} \mathrm{K})$. This value is higher than the experimental measurement of $44 \mathrm{~W} /(\mathrm{m} \mathrm{K})$, but the difference is within the discrepancies between the experimental data and modeling predictions for the solid films shown in Fig. 2.

In addition to the structure described earlier, Tang et al. also measured the thermal conductivities of 100 -nm-thick nanoporous films with porosity of 0.35 and average pitch/neck of $350 / 152 \mathrm{~nm}$ and 140/59 nm. Inspection of Figs. 2(a) and 2(b) in their paper shows large variability in the pore size, shape, and spacing. Furthermore, the second of these films has a limiting dimension of $59 \mathrm{~nm}$, which is close to where the solid film experimental data deviates from the modeling predictions (see our Fig. 2). As such, we do not believe that the free path sampling technique, which assumes a perfect periodicity of the pores, is appropriate for predicting the thermal conductivity of these two structures.

\section{Cross-plane direction}

Measured $^{3}$ and predicted cross-plane thermal conductivities are plotted in Fig. 3(b). As with the in-plane heat flow, the 
modeling results show a decrease in thermal conductivity with increasing porosity. The experimental results, however, are an order of magnitude smaller than the modeling predictions and show no significant porosity dependence. From calculations on a large number of films, we find that the predicted ratio of the in-plane to cross plane thermal conductivities varies linearly with porosity from $1.2(\phi=0.07)$ to $0.82(\phi=$ $0.38)$. Moreover, the predicted solid film thermal conductivity (plotted at $\phi=0$ ) is almost twice as high as that measured in the experiments.

\section{Thermal conductivity accumulation}

Recent experimental measurements suggest the possibility of resolving the contribution of phonons with different mean free paths to thermal conductivity. ${ }^{45-49}$ This information could be used to formulate strategies for reducing thermal conductivity by selectively scattering specific phonon modes using defects, grain boundaries, and surfaces. The thermal conductivity accumulation functions ${ }^{50,51}$ for bulk silicon and solid silicon films with thicknesses of 50, 100, 500, and $1000 \mathrm{~nm}$ (in-plane direction) are plotted in Figs. 4(a) and 4(b). The vertical coordinate of any point on the accumulation function represents the thermal conductivity that comes from phonons with mean free path less than the horizontal coordinate of that point. In Fig. 4(a), the bulk mean free path is used to plot the accumulation functions, while the mean free paths in the structure of interest are used in Fig. 4(b).

By plotting against the bulk mean free path in Fig. 4(a), we see where phonons start to be affected by boundary scattering. For all films, deviations in the accumulation functions from bulk occur at bulk mean free paths smaller than the film thicknesses (e.g., around $100 \mathrm{~nm}$ for the 500-nm-thick film). This result makes sense because phonons can originate anywhere in the film. It is also important to note that most phonons do not travel purely in the cross-plane direction. As such, depending on how its group velocity vector is oriented compared to the film, each phonon mode will start to be affected at a different film thickness.

When plotted vs the film mean free path in Fig. 4(b), the accumulation functions shift to the left as there are more phonon modes with smaller mean free paths. For the 50- and $100-\mathrm{nm}$ films, the accumulation functions rise more quickly than the bulk curve starting at a mean free path of $20 \mathrm{~nm}$. The film accumulation functions must then cross the bulk curve (at 70 and $95 \mathrm{~nm}$ ) as their total thermal conductivities are smaller. For the 500-nm film, the mean free path reductions cause the film accumulation function to follow the bulk curve up to a mean free path of $400 \mathrm{~nm}$. It then rises above the bulk curve, crossing back over at $500 \mathrm{~nm}$. For the 1000-nm film, the film accumulation function rises above the bulk curve at $600 \mathrm{~nm}$, then crosses it at $1275 \mathrm{~nm}$. It interesting to note that the crossovers occur close to the film thicknesses.

As discussed in Sec. IV B, the length scale used in the Matthiessen rule for a solid film is related to its thickness [Eq. (4)]. The results shown in Fig. 4(b) suggest that this length scale can be estimated from the accumulation function from the crossover mean free path. Extending this idea, we can extract equivalent film thicknesses $L_{\mathrm{eq}}$ for nanoporous films from their accumulation functions. The accumulation
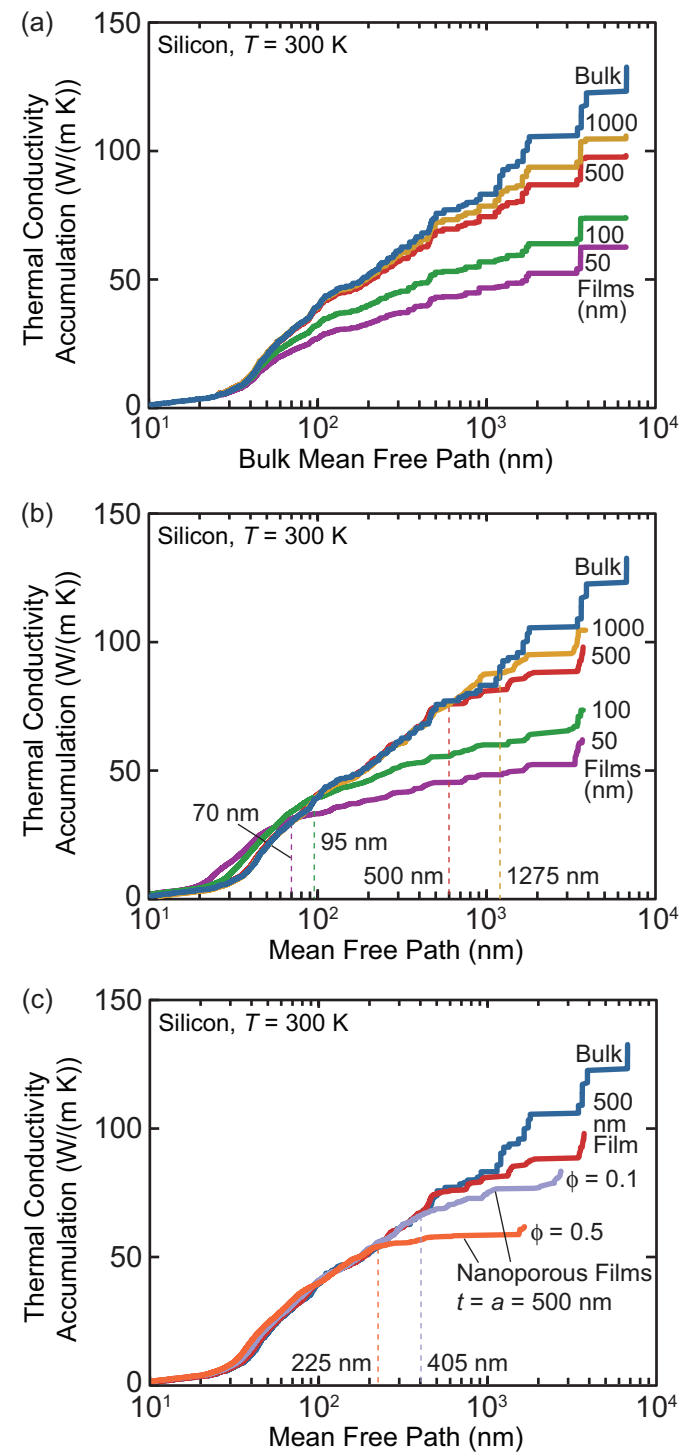

FIG. 4. (Color online) Thermal conductivity accumulation functions for (a) and (b) bulk silicon and solid silicon films, and (c) bulk silicon, a 500-nm-thick solid film, and two 500-nm-thick periodic nanoporous films with pore pitch of $500 \mathrm{~nm}$ and porosities of 0.1 and 0.5 . The bulk mean free paths are used to generate the curves in (a) while the mean free paths in the structure of interest are used to generate the curves in (b) and (c). All film accumulation functions are for the in-plane direction.

functions for two 500-nm-thick nanoporous silicon films with a pore pitch of $500 \mathrm{~nm}$ and porosities of 0.1 and 0.5 are plotted in Fig. 4(c), along with the bulk curve and that for a solid film of the same thickness. The accumulation functions for the nanoporous films follow the bulk and solid film curves closely up a certain point, after which they level off.

The equivalent film thickness for the two nanoporous films included in Fig. 4(c) and three more with different porosities are provided in Table I. The thermal conductivities predicted using these length scales in Eq. (4) with the Matthiessen rule are also provided in Table I along with those predicted from the free path sampling technique. We note that using the minimum feature size (i.e., the smaller of the pore neck and the film thickness) as the length scale in the Matthiessen rule ${ }^{23}$ leads to 
TABLE I. Equivalent film thickness extracted from thermal conductivity accumulation function [see Fig. 4(c)] and in-plane thermal conductivity predicted using the Matthiessen rule and Eq. (4) for nanoporous films. The pore pitch and film thickness are $500 \mathrm{~nm}$ for all structures.

\begin{tabular}{lccc}
\hline \hline & $\begin{array}{c}L_{\mathrm{eq}} \\
(\mathrm{nm})\end{array}$ & $\begin{array}{c}k\left(\text { Matthiessen rule w/ } L_{\mathrm{eq}}\right) \\
(\mathrm{W} /(\mathrm{m} \mathrm{K}))\end{array}$ & $\begin{array}{c}k \text { (free path sampling) } \\
(\mathrm{W} /(\mathrm{m} \mathrm{K}))\end{array}$ \\
\hline 0.1 & 405 & 91 & 84 \\
0.2 & 365 & 89 & 78 \\
0.3 & 275 & 85 & 72 \\
0.4 & 270 & 85 & 67 \\
0.5 & 225 & 82 & 62 \\
\hline \hline
\end{tabular}

an isotropic thermal conductivity and so we do not pursue that direction. The relative error between the thermal conductivities predicted from free path sampling and the Matthiessen rule using the equivalent film thickness increases as the porosity increases, reaching a maximum value of $32 \%$. This result reinforces the need to use the free path sampling technique for structures with multiple feature sizes.

\section{DISCUSSION}

The term "coherent phonon mode" has been used to describe vibrational modes that emerge when a secondary periodicity is added to a system (e.g., the layers in a superlattice or the pores in a periodic nanoporous film)..$^{1,3,5,23,52-55}$ It has been argued that these phonons affect thermal transport in periodic nanoporous films by modifying the dispersion curves and/or by changing the nature of the phonon scattering. $1,3,5,23$

Coherence implies the interference of waves, either constructively or destructively. In a periodic nanoporous film, coherent phonon modes will emerge if (i) the phonons scatter specularly off the pores, maintaining information about their phase, and (ii) these phonons do not scatter with other phonons between the pore collisions, allowing for interference to develop. Specular scattering requires that the scale of the surface roughness be much smaller than the phonon wavelengths that contribute to thermal transport. An absence of scattering is a good approximation for light (photons) and a reasonable approximation for phonons at acoustic frequencies (i.e., $\mathrm{kHz}$ to $\mathrm{MHz}$ ). For thermal phonons, which have $\mathrm{THz}$ frequencies, very low phonon-phonon scattering rates require a lack of scattering channels. Such a condition may be found at low temperatures, in materials with a large phonon band gap or reduced dimensionality, or in nanostructures with feature sizes much smaller than the bulk mean free paths.

As discussed in Sec. III, we expect that phonon-pore scattering at a temperature of $300 \mathrm{~K}$ will be primarily diffuse. Furthermore, as shown in Fig. 4(a), the majority of the bulk mean free paths in silicon at a temperature of $300 \mathrm{~K}$ are comparable to or smaller than the feature sizes of the nanoporous films studied by El Kady et al. and Hopkins et al. Based on these two arguments, it is unlikely that coherent phonon effects are important in those structures. This statement is supported by the agreement of the predictions of the free path sampling technique (which considers only bulk-like phonons and their interactions with each other and system boundaries) with the in-plane experimental measurements, as shown in Fig. 3(a)

That being said, it is worthwhile to discuss the conditions under which coherent phonon effects may emerge and play a role in thermal transport. Generally speaking, for a secondary periodicity to affect thermal transport, it should be on a length scale comparable to wavelengths of the phonons that contribute to thermal transport. In bulk silicon at room temperature, the phonons that dominate thermal transport have wavelengths of $1-5 \mathrm{~nm},{ }^{15}$ two orders of magnitude smaller than the feature sizes of the films studied by Hopkins et al. and El-Kady et al. Luckyanova et al. recently reported thermal conductivity measurements on multilayer GaAs/AlAs structures with layer thickness $12 \mathrm{~nm}$ that indicate the increasing importance of phonons related to the secondary periodicity as temperature decreases from 150 to $30 \mathrm{~K} .^{55}$

In addition to a feature size that aligns with the dominant phonon wavelengths, there is a second important criterion for the emergence of coherent phonon effects: the quality of the periodicity. Consider the $\mathrm{Si} / \mathrm{Ge}$ superlattices studied by Landry and McGaughey using MD simulations, which have period lengths between 3 and $12 \mathrm{~nm} .{ }^{56}$ They predicted the period-length dependence of the thermal conductivity of superlattices with perfect interfaces and with interfaces where $12 \%$ of the atoms were switched in the layers next to the interfaces. The superlattices with perfect interfaces show an initially decreasing thermal conductivity with increasing period length that levels off, a trend consistent with the secondary periodicity affecting thermal transport. ${ }^{57-59}$ The results are completely different for the superlattices with interfacial species mixing. At the smallest period length, the thermal conductivity is almost an order of magnitude smaller than that for the perfect interfaces and increases with increasing period length. This trend is related to phonon-interface scattering. As the period length increases, the interface density decreases, and thermal conductivity increases. The key observation here is that a small amount of disorder destroyed the coherent effects. When mixing is present, the phonon modes are defined within each layer, but not across the interfaces. In the majority of experimental superlattice samples (except systems such as GaAs/AlAs superlattices, ${ }^{55}$ where epitaxial layers can be grown) such disorder will always be present. It thus seems unlikely that coherent effects can explain experimental superlattice thermal conductivity measurements, particularly at room temperature.

A similar argument can be made when considering the nanoporous films of Yu et al. and Tang et al. which have pore pitches of 34 and $55 \mathrm{~nm}$. Their structures show a strong variability in pore size, shape, and spacing. It therefore seems unlikely that coherent phonon effects will emerge. Only at very low temperatures, where the dominant phonon wavelengths become comparable to the pore pitch, might such effects be relevant.

How then to interpret the very low thermal conductivity measurements of Yu et al. and Tang et al.? As suggested by the modeling work of $\mathrm{He}$ et al., ${ }^{21}$ the thermal conductivity reduction may be a result of the emergence of nonpropagating vibrational modes in confined geometries. Such a mechanism could be present in rough silicon nanowires, which also have 
very low thermal conductivities. ${ }^{60,61}$ Further work in this direction is warranted.

\section{SUMMARY}

We used bulk silicon phonon properties obtained from forces calculated from DFT and the free path sampling technique to predict the thermal conductivities of solid and periodic nanoporous films. There is ambiguity in the application of the Matthiessen rule to the nanoporous films due to their multiple feature sizes. The free path sampling technique treats the phonons as particles with bulk properties that scatter with the pore boundaries. No effects of the secondary periodicity are included other than the geometry. Our in-plane thermal conductivity predictions are in good agreement with the experimental measurements of El-Kady et al. for periodic nanoporous films with feature sizes greater than $100 \mathrm{~nm}$.
This result indicates that coherent phonon effects in these films do not affect thermal transport. At this time, we are not able to explain the cross-plane thermal conductivities measured by Hopkins et al. on films similar to those studied by El-Kady et al.

\section{ACKNOWLEDGMENTS}

This work was supported by AFOSR Award No. FA95501010098 (A.J., A.J.H.M.) and the University of Texas at Austin (A.J.H.M.). We thank K. Esfarjani (Massachusetts Institute of Technology) for supplying the bulk phonon properties. We thank I. El-Kady (Sandia National Laboratory), P. E. Hopkins (University of Virginia), J. A. Malen (Carnegie Mellon University), and D. P. Sellan (The University of Texas at Austin) for their helpful discussions. We thank W.-L. Ong (Carnegie Mellon University) for his help with the figures. *mcgaughey@cmu.edu

${ }^{1}$ J.-K. Yu, S. Mitrovic, D. Tham, J. Varghese, and J. R. Heath, Nat. Nano 5, 718 (2010).

${ }^{2}$ J. Tang, H.-T. Wang, D. H. Lee, M. Fardy, Z. Huo, T. P. Russell, and P. Yang, Nano Lett. 10, 4279 (2010).

${ }^{3}$ P. E. Hopkins, C. M. Reinke, M. F. Su, R. H. Olsson, III, E. A. Shaner, Z. C. Leseman, J. R. Serrano, L. M. Phinney, and I. El-Kady, Nano Lett. 11, 107 (2011).

${ }^{4}$ J.-H. Lee, G. A. Galli, and J. C. Grossman, Nano Lett. 8, 3750 (2008).

${ }^{5}$ E. Dechaumphai and R. Chen, J. Appl. Phys. 111, 073508 (2012).

${ }^{6}$ Q. Hao, G. Chen, and M.-S. Jeng, J. Appl. Phys. 106, 114321 (2009).

${ }^{7}$ I. El-Kady, R. H. Olsson III, P. E. Hopkins, Z. C. Leseman, D. F. Goettler, B. Kim, C. M. Reinke, and M. F. Su, Phonon Manipulation with Phononic Crystals, Progress report SAND2012-0127 (Sandia National Laboratories, California, CA, 2012).

${ }^{8}$ A. J. H. McGaughey, E. S. Landry, D. P. Sellan, and C. H. Amon, Appl. Phys. Lett. 99, 131904 (2011).

${ }^{9}$ M. Asheghi, Y. K. Leung, S. S. Wong, and K. E. Goodson, Appl. Phys. Lett. 71, 1798 (1997).

${ }^{10}$ Y. S. Ju and K. E. Goodson, Appl. Phys. Lett. 74, 3005 (1999).

${ }^{11}$ D. Li, Y. Wu, P. Kim, L. Shi, P. Yang, and A. Majumdar, Appl. Phys. Lett. 83, 2934 (2003).

${ }^{12}$ P. Chantrenne, J. L. Barrat, X. Blase, and J. D. Gale, J. Appl. Phys. 97, 104318 (2005).

${ }^{13}$ A. J. H. McGaughey and A. Jain, Appl. Phys. Lett. 100, 061911 (2012).

${ }^{14}$ K. Esfarjani and H. T. Stokes, Phys. Rev. B 77, 144112 (2008).

${ }^{15}$ K. Esfarjani, G. Chen, and H. T. Stokes, Phys. Rev. B 84, 085204 (2011).

${ }^{16}$ R. Yang, G. Chen, and M. S. Dresselhaus, Phys. Rev. B 72, 125418 (2005).

${ }^{17}$ R. Prasher, J. Appl. Phys. 100, 034307 (2006).

${ }^{18}$ G. Romano, A. D. Carlo, and J. C. Grossman, J. Comput. Electron. 11, 8 (2012).

${ }^{19}$ A. S. Henry and G. Chen, J. Comput.: Theor. Nanosci. 5, 141 (2008).
${ }^{20}$ D. P. Sellan, E. S. Landry, J. E. Turney, A. J. H. McGaughey, and C. H. Amon, Phys. Rev. B 81, 214305 (2010).

${ }^{21}$ Y. He, D. Donadio, J.-H. Lee, J. C. Grossman, and G. Galli, ACS Nano 5, 1839 (2011).

${ }^{22}$ J.-H. Lee, J. C. Grossman, J. Reed, and G. Galli, Appl. Phys. Lett. 91, 223110 (2007).

${ }^{23}$ C. M. Reinke, M. F. Su, B. L. Davis, B. Kim, M. I. Hussein, Z. C. Leseman, R. H. Olsson-III, and I. El-Kady, AIP Adv. 1, 041403 (2011).

${ }^{24}$ D. C. Wallace, Thermodynamics of Crystals (Cambridge University Press, Cambridge, UK, 1972).

${ }^{25}$ A. A. Maradudin and A. E. Fein, Phys. Rev. 128, 2589 (1962).

${ }^{26} \mathrm{M}$. T. Dove, Introduction to Lattice Dynamics (Cambridge University Press, Cambridge, UK, 1993).

${ }^{27}$ J. E. Turney, E. S. Landry, A. J. H. McGaughey, and C. H. Amon, Phys. Rev. B 79, 064301 (2009).

${ }^{28}$ G. P. Srivastava, The Physics of Phonons (Adam Hilger, Bristol, 1990).

${ }^{29}$ J. M. Ziman, Electrons and Phonons (Oxford, New York, 2001).

${ }^{30}$ H. B. G. Casimir, Physica 5, 495 (1938).

${ }^{31}$ J. E. Turney, A. J. H. McGaughey, and C. H. Amon, J. Appl. Phys. 107, 024317 (2010).

${ }^{32}$ J.-P. M. Péraud and N. G. Hadjiconstantinou, Phys. Rev. B 84, 205331 (2011)

${ }^{33}$ J.-P. M. Péraud and N. G. Hadjiconstantinou, Appl. Phys. Lett. 101, 153114 (2012)

${ }^{34}$ J. C. Duda, T. E. Beechem, J. L. Smoyer, P. M. Norris, and P. E. Hopkins, J. Appl. Phys. 108, 073515 (2010).

${ }^{35}$ R. Kremer, K. Graf, M. Cardona, G. Devyatykh, A. Gusev, A. Gibin, A. Inyushkin, A. Taldenkov, and H.-J. Pohl, Solid State Commun. 131, 499 (2004).

${ }^{36}$ M. Asheghi, K. Kurabayashi, R. Kasnavi, and K. E. Goodson, J. Appl. Phys. 91, 5079 (2002).

${ }^{37}$ A. D. McConnell and K. E. Goodson, Annu. Rev. Heat Transf. 14, 129 (2005).

${ }^{38}$ D. A. Broido, M. Maloney, G. Birner, N. Mingo, and D. Stewart, Appl. Phys. Lett. 91, 231922 (2007).

${ }^{39}$ D. Song and G. Chen, Appl. Phys. Lett. 84, 687 (2004). 
${ }^{40}$ Y. S. Ju, Appl. Phys. Lett. 87, 153106 (2005).

${ }^{41}$ W. Liu and M. Asheghi, J. Heat Transf. 128, 75 (2006).

${ }^{42}$ M. Kaviany, Heat Transfer Physics (Cambridge University Press, New York, 2008).

${ }^{43}$ Z. Hashin and S. Shtrikman, J. Appl. Phys. 33, 3125 (1962).

${ }^{44}$ Eucken, A. Forschung auf dem Gebiete des Ingenieurwesens; VDI-verlag g.m.b.h.: Dusseldorf, 1932, Ausgabe B, 3/4 VDI Forschungsheft 353.

${ }^{45}$ Y. K. Koh and D. G. Cahill, Phys. Rev. B 76, 075207 (2007).

${ }^{46}$ M. E. Siemens, Q. Li, R. Yang, K. A. Nelson, E. H. Anderson, M. M. Murnane, and H. C. Kapteyn, Nat. Mater. 9, 26 (2010).

${ }^{47}$ A. J. Minnich, J. A. Johnson, A. J. Schmidt, K. Esfarjani, M. S. Dresselhaus, K. A. Nelson, and G. Chen, Phys. Rev. Lett. 107, 095901 (2011).

${ }^{48}$ J. A. Johnson, A. A. Maznev, M. T. Bulsara, E. A. Fitzgerald, T. C. Harman, S. Calawa, C. J. Vineis, G. Turner, and K. A. Nelson, J. Appl. Phys. 111, 023503 (2012).

${ }^{49}$ K. T. Regner, D. P. Sellan, Z. Su, C. H. Amon, A. J. H. McGaughey, and J. A. Malen, Nat. Commun. 4, 1640 (2013).

${ }^{50} \mathrm{C}$. Dames and G. Chen, in Thermoelectrics Handbook: Macro to Nano, edited by D. M. Rowe (Taylor \& Francis, Boca Raton, 2006) pp. 42-1-42-11.
${ }^{51}$ F. Yang and C. Dames, Phys. Rev. B 87, 035437 (2013).

${ }^{52}$ A. Yamamoto, T. Mishina, Y. Masumoto, and M. Nakayama, Phys. Rev. Lett. 73, 740 (1994).

${ }^{53}$ Y. Ezzahri, S. Grauby, J. M. Rampnoux, H. Michel, G. Pernot, W. Claeys, S. Dilhaire, C. Rossignol, G. Zeng, and A. Shakouri, Phys. Rev. B 75, 195309 (2007).

${ }^{54}$ B. Yang and G. Chen, Phys. Rev. B 67, 195311 (2003).

${ }^{55}$ M. N. Luckyanova, J. Garg, K. Esfarjani, A. Jandl, M. T. Bulsara, A. J. Schmidt, A. J. Minnich, S. Chen, M. S. Dresselhaus, Z. Ren, E. A. Fitzgerald, and G. Chen, Science 338, 936 (2012).

${ }^{56}$ E. S. Landry and A. J. H. McGaughey, Phys. Rev. B 79, 075316 (2009).

${ }^{57}$ M. V. Simkin and G. D. Mahan, Phys. Rev. Lett. 84, 927 (2000).

${ }^{58}$ Y. Chen, D. Li, J. R. Lukes, Z. Ni, and M. Chen, Phys. Rev. B 72, 174302 (2005)

${ }^{59}$ E. S. Landry, M. I. Hussein, and A. J. H. McGaughey, Phys. Rev. B 77, 184302 (2008).

${ }^{60}$ A. I. Boukai, Y. Bunimovich, J. Tahi-Kheli, J.-K. Yu, W. A. G. Goddard, and J. R. Heath, Nature (London) 451, 168 (2008).

${ }^{61}$ A. I. Hochbaum, R. Chen, R. D. Delgado, W. Liang, E. C. Garnett, M. Najarian, A. Majumdar, and P. Yang, Nature (London) 451, 163 (2008). 